\title{
Ultrasound-Guided Infraclavicular Axillary Vein Cannulation
}

\author{
Miguel A. García-Díaz, Manuel Ruiz-Castro \\ Department of Anesthesia, University Hospital Príncipe de Asturias, Madrid, Spain \\ Email:mruizc@hotmail.com
}

How to cite this paper: García-Díaz, M.A. and Ruiz-Castro, M. (2017) UltrasoundGuided Infraclavicular Axillary Vein Cannulation. International Journal of Clinical Medicine, 8, 227-235.

https://doi.org/10.4236/ijcm.2017.84022

Received: January 24, 2017

Accepted: April 27, 2017

Published: April 30, 2017

Copyright $\odot 2017$ by authors and Scientific Research Publishing Inc. This work is licensed under the Creative Commons Attribution International License (CC BY 4.0).

http://creativecommons.org/licenses/by/4.0/

(c) (i) Open Access

\begin{abstract}
Background and Objective: Percutaneous central venous cannulation is a common invasive procedure. In comparison with an external landmark technique, the advantages of ultrasound-guided venous access include direct visualization of the anatomy and in vivo visualization of venous cannulation. Methods: We evaluated an ultrasound-guided technique for infraclavicular axillary vein cannulation, focusing on its ease of use, success rate and complications rate. One hundred and twenty patients who submitted to central venous catheter placement were punctured using our technique. The patients were positioned so that their ipsilateral upper limb was abducted at $90^{\circ}$ to the longitudinal axis, which makes it possible to visualize the infraclavicular vessels due to the elevation of the clavicle, thereby improving accessibility. Results: Cannulation was successful in all patients. The median time from the start of the first puncture (of the skin) until the aspiration of blood was $15 \mathrm{~s}$ (range 7 - $135 \mathrm{~s}$ ). Both infraclavicular axillary veins were cannulated, and the vein was punctured successfully at the first attempt in $95 \%$ of the patients, without complications during the procedure. Conclusion: We propose an ultrasound-guided infraclavicular approach of the axillary vein, with a high success rate and no complications in the present cohort.
\end{abstract}

\section{Keywords}

Ultrasonography, Catheterization, Central Venous, Central Venous Catheter, Ultrasound-Guided Technique, Infraclavicular Axillary Vein

\section{Introduction}

Central venous catheterization (CVC) is a common invasive procedure. Complications associated with CVC are often related to blind passage of the needle, and include bleeding, arterial puncture, pneumothorax, nerve damage, pain and 
other less common complications.

Most authors [1] [2] [3] [4] [5], currently recommend the use of ultrasound (US) for placing catheters in adults. In comparison with the use of superficial anatomical landmarks, the advantages of US-guided venous access include direct visualization of the anatomy and the in vivo visualization of venous cannulation [6] [7]. The use of real-time US technology to guide needle insertion has improved success rates and reduced the incidence of complications [1] [2].

It has recently been shown that US is beneficial for catheterizing the internal jugular vein (IJV), as well as the axillary and mid-arm basilica and cephalic veins. The IJV is more popular due to the easier access and perceived lower risk of complications, however, subclavian vein (SCV) catheter placement is associated with a lower incidence of infection and thrombosis [8] [9] [10].

Most authors contend that infraclavicular approaches are difficult to visualize with US due to the SCV being located under the clavicle and the inability to place the linear array probe in a position that allows the vessel to be visualized. For this reason, US-guided SCV access is often described using a supraclavicular [8], or a distal approach.

We have developed an US-guided infraclavicular approach for axillary catheter placement using a standard high-frequency linear probe. The location of the axillary vein approach is very proximal, close to, or even over the lip of the first rib, where the SCV is renamed, and hence this approach could be comparable to the approach of the SCV.

\section{Method}

Ethical approval for this prospective study (protocol number: PI/422010), was provided by the Ethical Committee of the Príncipe de Asturias University Hospital, Alcalá de Henares, Madrid, Spain (chairperson: Prof. Elvira Poves Martínez) on 25 November 2010. Written informed consent to participate was obtained from each patient.

A GE Healthcare Venue 40 devices and a GE 12L-SC multifrequency linear transducer at $5-13 \mathrm{MHz}$ (GE Healthcare, a division of General Electric Company, Piscataway, New Jersey, USA) were used. Both authors performed this procedure in sixty patients each. The patients were assigned to the study in a consecutive way. The objective of this work was to make a purely descriptive study, so it was not considered to perform the comparison with the conventional technique without ultrasonography, given the high success rate in the US-guided puncture. Therefore, the size of the sample was intended to be representative, although we did not perform a calculation of the sample size when not having to observe differences with a control group.

The position of the patient and the anesthesiologist varies depending upon the standard external-anatomical-landmark-guided catheterization technique. The patient should be positioned so that the ipsilateral upper limb is abducted at $90^{\circ}$ to the longitudinal axis, which makes it possible to visualize the infraclavicular vessels due to the elevation of the clavicle, thereby improving accessibility. This 
improves the US visualization of the infraclavicular axillary vein (IAV), making almost its entire length visible. If the probe is moved laterally, it is possible to visualize the course of the axillary vein, while if the probe is moved medially with a slight cranial angulation, it is possible to visualize SCV.

Moreover, placing the patient in the Trendelenburg position increases venous engorgement and ease of viewing.

The anesthesiologist puts the patient's head and locates the sterile field and the equipment needed for the cannulation cephalad to the patient's arm or beside it, allowing intraoperative puncture. The anesthesiologist's non-dominant hand holds the US probe, while the dominant hand performs the cannulation.

Initial exploration of the area with US is essential for placing the probe infraclavicularly, parallel and in close contact with the bottom edge of the clavicle, in the infraclavicular fossa, just lateral to the clavicular head of the sternocleidomastoid muscle Figure 1.

A slight cranial angulation of the probe will facilitate the visualization of the infraclavicular vessels, which will be visible as long as the probe is moving parallel to the clavicle, medially and laterally. The vessels on the left side are caudal to those on the right side and the required degree of cranial angulation of the US probe is lower on the left side.

The image obtained from the IAV is medial and superficial to the infraclavicular axillary artery (IAA), and has an elongated shape, close to the long axis view Figure 2.

Furthermore, the pressure of the US probe on the patient can partially collapse the vein. In US imaging, the IAA is normally viewed in cross section, and it appears rounded, pulsatile, and incompressible under the pressure of the US probe.

In-plane venipuncture is achieved from the lateral end of the probe, in the same plane as the US beam, which displays the progress of the needle as it moves

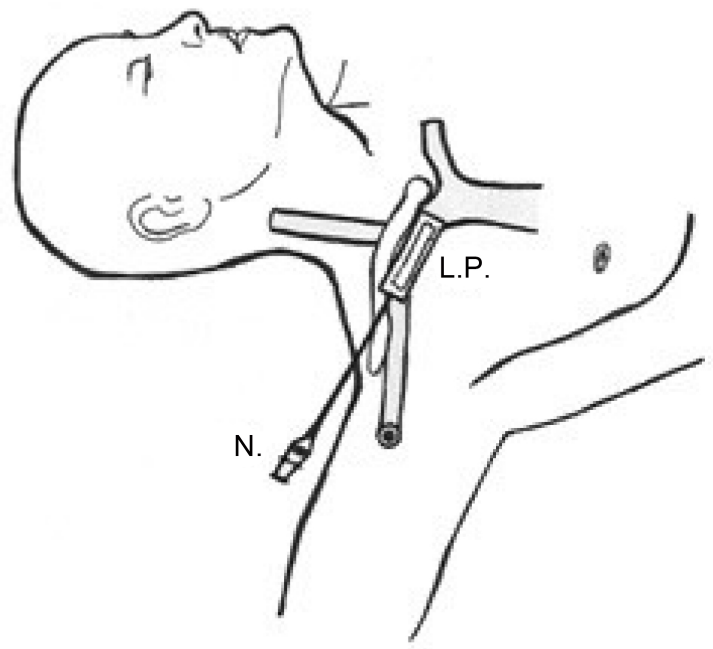

Figure 1. Artistic drawing of the location and placement of the linear array probe (LP) and the needle (N) during the infraclavicular ultrasound-guided approach to cannulation of the right axillary vein. 


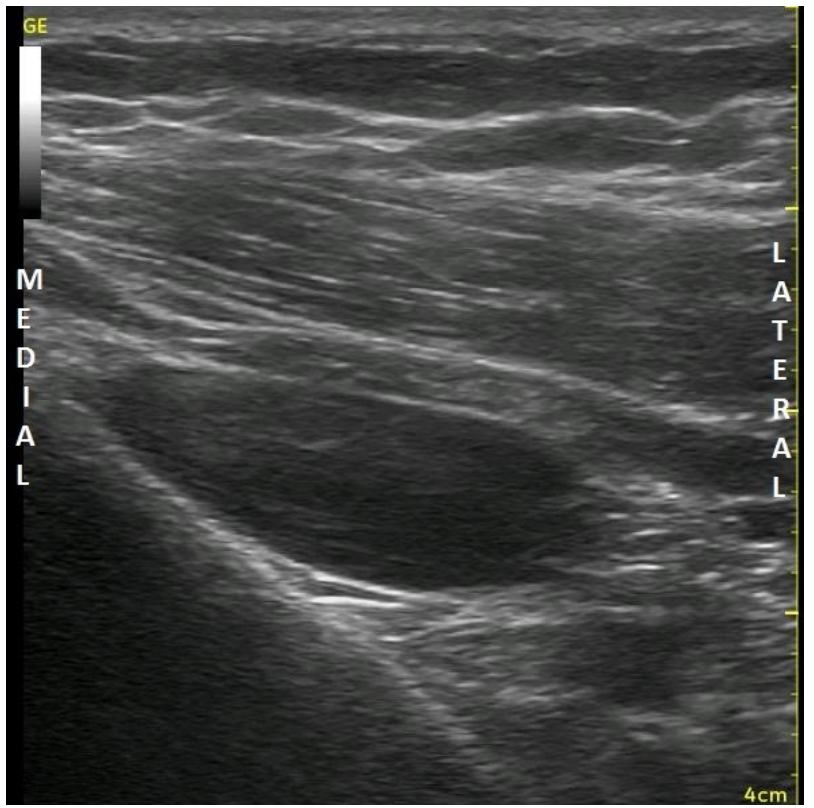

(a)

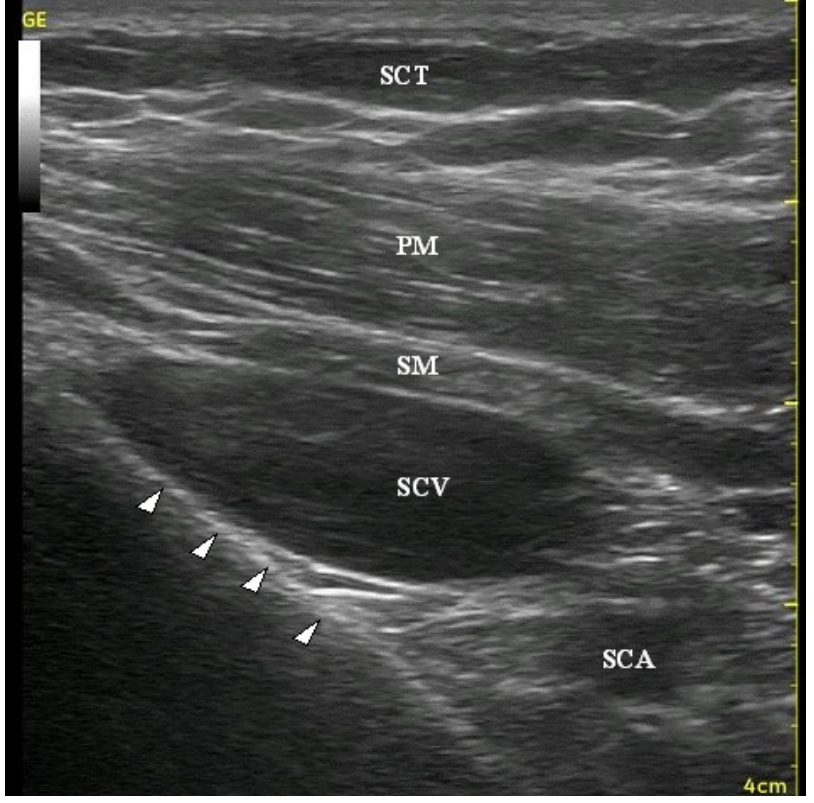

(b)

Figure 2. Ultrasonographic image obtained at the infraclavicular level. (a) Blank image. (b) Labelled image. SCT, subcutaneous cellular tissue; PM, pectoralis major muscle; SM, subclavian muscle; SCV, subclavian vein; SCA, subclavian artery; Arrowheads, first rib.

into the lumen of the vein. It is of paramount importance that the operator observes the needle advancement in real time. To improve visualization of the puncture needle, it is helpful to exert a slight pressure on the inner edge of the US probe so that the angle between the US beams and the needle is as close as possible to $90^{\circ}$.

While inserting the needle, mild aspiration is performed with the syringe, so 
that blood is observed entering the syringe when the tip of the needle reaches the vessel lumen. Real-time visualization of the extent of the venous lumen demonstrates that the needle tip is at the correct location.

The venous wall is more elastic in younger patients, and so the tip of the needle can be pushed against the wall without perforating it. Aspiration of blood will be necessary to show that the needle tip has reached the vein lumen, as the progress of the needle tip will be visible on the US image. Only minimal further progression from this point will cause the needle tip to enter the lumen without piercing the posterior venous wall.

The usual Seldinger procedure is then performed. The no dominant hand releases the US probe and holds the needle. The guide wire is introduced into the venous lumen without a real-time US view. Once the guide wire is in the vein, its position is rechecked with the probe, and the supraclavicular area is scanned to detect possible migration of the guide wire into the IJV Figure 3.

Early possible complications such as pneumothorax or hematoma, as well as catheter-tip position are checked after CVC positioning in every patient by means of a chest $\mathrm{x}$-ray.

\section{Results}

For a one year period, 120 patients who submitted to CVC were punctured using our technique. In all except one case the IAVs could be easily visualized from the infraclavicular level, so no patients were excluded from the study. The demographic data of the patients are listed in Table 1.

A single attempt was required in $95 \%$ of the cases (114 patients). A second attempt was needed in three cases, two of them because although the IAV was catheterized at the first attempt, the vein was "lost" during guide-wire manipulation, thus requiring a second IAV puncture. In another case a chest $\mathrm{x}$-ray revealed that the catheter had migrated distally to the distal axillary vein, which made it necessary to remove the catheter and puncture again on the other side. In one IAV the venous lumen on the left side could not be seen because it was thrombosed, but it could be catheterized on the other side. Another guide wire that migrated into the IJV could be repositioned without a new puncture, partially removing the guide wire, and reintroducing it under US guidance. The mean number of attempts per patient was 1.05. More than two attempts at venipuncture, which is known to increase the incidence of complications, were not needed in the present cohort.

Venous hematomas due to multiple failed attempts to catheterize the vein were thus not seen in this series. These results are comparable with those achieved using US-guided techniques for IJV puncture.

The right IAV was the most frequently cannulated vein (55\%), with most of the cannulations carried out during surgery, but no more attempts were needed when the left IAV was punctured. The median time from the start of puncture to aspiration of blood was $15 \mathrm{~s}$ (range 7 - $135 \mathrm{~s}$ ). We experienced no arterial puncture and no pneumothorax in this series, even though this cohort represented 


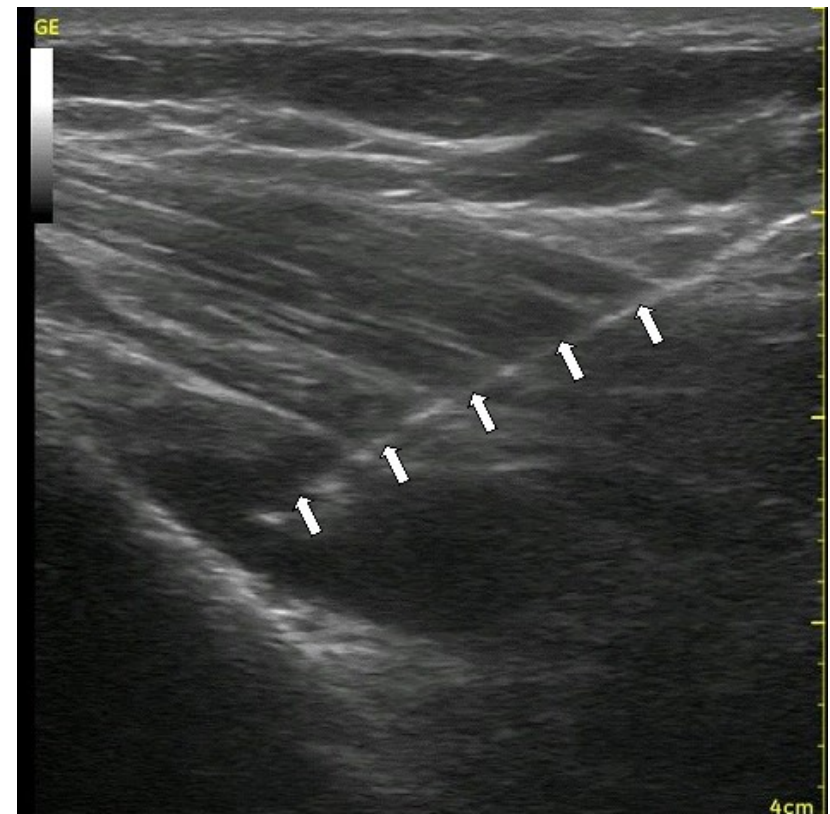

(a)

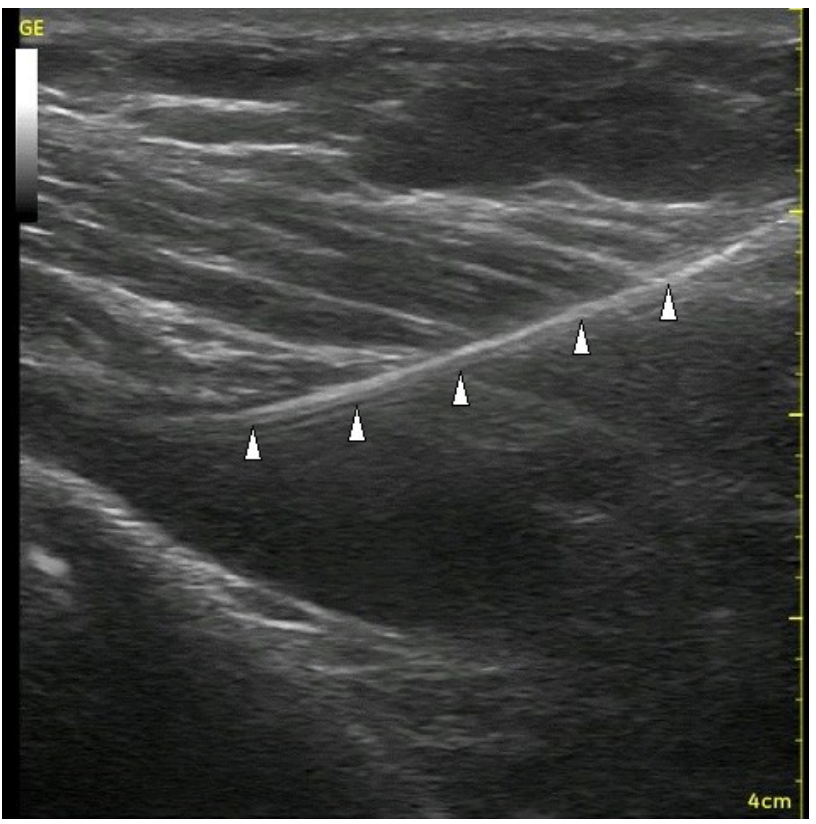

(b)

Figure 3. Puncture technique. (a) The needle punctures the IAV and progresses under direct vision to the lumen of the vein. Arrows, needle. (b) The guide-wire is inserted after removing the needle. Arrowheads, guide-wire.

Table 1. Demographic distribution of patients undergoing US-guided infraclavicular axillary vein cannulation.

\begin{tabular}{ccccc}
\hline Mean & Age & Weight & Height & Body Mass Index \\
\hline & 66.93 years & $74.60 \mathrm{Kg}$ & $164.43 \mathrm{~cm}$ & $27.56 \mathrm{Kg} / \mathrm{cm}^{2}$ \\
Standard Deviation & 13.94 years & $10.66 \mathrm{Kg}$ & $8.60 \mathrm{~cm}$ & $3.12 \mathrm{Kg} / \mathrm{m}^{2}$ \\
\hline
\end{tabular}

${ }^{*}$ Total number of cases 120: 68 females and 52 males. 
our learning curve for this technique.

\section{Discussion}

US-guided venous access has been described and widely recommended for IJV cannulation [11]. However, few studies have investigated US-guided SCV puncture, most of which have focused on paediatric venous cannulation [6] [7]. One paper reports the use of US to puncture the SCV via a supraclavicular approach with an endocavitary probe with good results [8], but we describe here a technique that uses a standard linear array probe to puncture the IAV, in close proximity to the SCV. The boundary between the two is defined by the edge of the first rib, so we believe that there are no clinically significant differences.

In previous studies, the venous approach was performed more laterally, even at the level of the mid-axillary vein, where mobility is greater, but fixation of the catheter is worse.

Only a few articles [12] [13] have reported on US-guided for axillary vein puncture. Two studies imaged both the vein and the needle transversely, which offers poorer needle visualization. Another paper [14] describes a more lateral approach to the axillary vein, where longitudinal visualization of both the vein and needle was possible. Our longitudinal in-line puncture technique offers a clear view of both the needle and the vein, which should produce consistently good results. The risk of accidental puncture of pleura or other structures (e.g., arteries) is lower, regardless of how deep the needle is, since the advancement of the needle, the vessel, and the surrounding structures can all be viewed in real time. Moreover, the place where the SCV passes over the first rib has been defined as a safe spot for its cannulation, since at this location, the first rib protects the lung from accidental puncture and the direction of the subclavian artery differs from that of the SCV, passing behind the anterior scalene muscle whereas the vein passes in front of it.

Abduction of the upper limb and the clavicle with it provides a US view of the infraclavicular vessels at this level, allowing safe puncture and cannulation.

Puncture time was measured in this study. The preparation time is known to be longer with US-guided techniques than with landmark-guided techniques. With experience, anatomical screening, and appropriate equipment preparation, the time should not exceed $5 \mathrm{~min}$. Because the anatomical (vein position) and pathological (vein thrombosis) variations are now diagnosed by US, the time from skin puncture to guide-wire placement is reduced.

Furthermore, the very time-consuming vicious circle of multiple attempts, venous or arterial hematoma, failed puncture, and need to change to another central venous insertion site is probably minimized by US guidance.

The entire procedure is likely to be completed faster than when using the anatomical-landmark-based technique. It should also be noted that the procedure can be carried out during surgery because the abducted position of the upper extremities is common in the operating room, thus further reducing the time required. This is a clear advantage over the traditional external landmark technique where the adducted position of the upper extremity is required and forces the anesthesi- 
ologist to cannulate the IJV in the operating setting, associated with a higher incidence of infection and thrombosis [8] [9] [10].

Another advantage of US is the early detection of incorrect guide-wire catheterization or migration of the guide wire into the IJV. In our study, we experienced a single case of misplacement of the guide-wire, perhaps because the abduction of the upper limb directs the IAV toward the territory of the superior vena cava.

In a completely sterile setting, the assistance of a third hand is usually not needed because the probe can be left in the sterile field during maneuvers that require two hands, such as clavicle palpation for needle insertion or guide-wire advancement.

The use of US not only provides needle guidance but also makes it easy to check the patency of the IAV, regardless of whether the cases are hypovolemic patients whose veins have little filler, or are well hydrated (e.g., with engorged veins). Lumen vein in hypovolemic patients is very narrow, being very difficult to place the tip of the needle inside. When it is closed to the venous wall, a Valsalva maneuver can distend the vein and facilitate the puncture.

The learning curve of this approach, as for every US-guided technique, will probably be highly operator-dependant. Prior to the start of the study, each of the anaesthesiologists cannulated at least three IAV with this US-guided technique, to familiarize themselves with it. Then, during the study, each of the anesthesiologists performed this procedure in sixty patients. Both of them had previous experience in US-guided puncture techniques (regional anesthesia and CVC) and in IJV and SCV anatomical-landmark-based cannulation.

We consider that it takes a minimum of five cases to perform the procedure safely, with many more being needed in the absence of prior experience in managing US. For inexperienced clinicians, residents, or fellows, the US-guided IAV cannulation should be considered an advanced technique given the complexity and risk of complications with this approach.

In conclusion US-guided IAV cannulation can be performed by using a linear-array probe and ipsilaterally abducting the patient's upper limb at $90^{\circ}$. We propose an infraclavicular approach when performing this technique, since it was associated with a high success rate and no complications in this the present cohort. This procedure is safe and easy to learn for CVC, and we recommend this technique even in an early learning stage. Moreover, the procedure can be performed in an intraoperative setting.

\section{Acknowledgements}

We thank M. Sanz, MD, F. Barrios and M. Ayuso for their assistance with the study. This work was supported by the Department of Anesthesiology, University Hospital Príncipe de Asturias, Alcalá de Henares, Madrid, Spain.

\section{References}

[1] Waldman, C.S. (2001) Use of Central Venous Catheters (CVC)-An Algorithm. 
Care of the Critically Ill, 17, 148-149.

[2] Randolph, A.G., Cook, D.J., Gonzales, C.A. and Pribble, C.G. (1996) Ultrasound Guidance for Placement of Central Venous Catheters: A Meta-Analysis of the Literature. Critical Care Medicine, 24, 2053-2058. https://doi.org/10.1097/00003246-199612000-00020

[3] Maslove, D. and Mihm, F. (2010) Ultrasound-Guided Internal Jugular Vein Cannulation. The New England Journal of Medicine, 363, 796. https://doi.org/10.1056/NEJMc1006062

[4] Levitov, A.B., Aziz, S. and Slonim, A.D. (2009) Before We Go Too Far: Ultrasound-Guided Central Catheter Placement. Critical Care Medicine, 37, 2473-2474. https://doi.org/10.1097/CCM.0b013e3181a9f694

[5] Barash, P. (2009) Ultrasound-Guided Central Venous Access. F1000 Medicine Reports, 1, 65. https://doi.org/10.3410/m1-65

[6] Hind, D., Calvert, N., McWilliams, R., et al. (2003) Ultrasonic Locating Devices for Central Venous Cannulation: Meta-Analysis. BMJ, 327, 361.

https://doi.org/10.1136/bmj.327.7411.361

[7] Theodoro, D., Bausano, B., Lewis, L., Evanoff, B. and Kollef. M. (2010) A Descriptive Comparison of Ultrasound-Guided Central Venous Cannulation of the Internal Jugular Vein to Landmark-Based Subclavian Vein Cannulation. Academic Emergency Medicine, 17, 416-422. https://doi.org/10.1111/j.1553-2712.2010.00703.x

[8] Mallin, M., Louis, H. and Madsen, T. (2010) A Novel Technique for Ultrasound-Guided Supraclavicular Subclavian Cannulation. American Journal of Emergency Medicine, 28, 969-969.

[9] McGee, D.C. and Gould, M.K. (2003) Preventing Complications of Central Venous Catheterization. The New England Journal of Medicine, 348, 1123-1133. https://doi.org/10.1056/NEJMra011883

[10] Stone, M.B., Moon, C., Sutijono, D. and Blaivas, M. (2010) Needle Tip Visualization during Ultrasound-Guided Vascular Access: Short-Axis vs. Long-Axis Approach. American Journal of Emergency Medicine, 28, 343-347.

[11] Gualtieri, E., Deppe, S.A., Sipperly, M.E., et al. (1995) Subclavian Venous Catheterization: Greater Success Rate for Less Experienced Operators Using Ultrasound Guidance. Critical Care Medicine, 23, 692. https://doi.org/10.1097/00003246-199504000-00018

[12] Sharma, A., Bodenham, A.R. and Mallick, A. (2004) Ultrasound-Guided Infraclavicular Axillary Vein Cannulation for Central Venous Access. British Journal of Anaesthesia, 93, 188-192. https://doi.org/10.1093/bja/aeh187

[13] Sandhu, N.S. (2004) Transpectoral Ultrasound-Guided Catheterization of the Axillary Vein: An Alternative of Standard Catheterization of the Subclavian Vein. Anesthesia \& Analgesia, 99, 183-187. https://doi.org/10.1213/01.ANE.0000117283.09234.2C

[14] Stone, M.B., Nagdev, A., Murphy, M.C. and Sisson, C.A. (2010) Ultrasound Detection of Guidewire Position during Central Venous Catheterization. American Journal of Emergency Medicine, 28, 82-84. 
Submit or recommend next manuscript to SCIRP and we will provide best service for you:

Accepting pre-submission inquiries through Email, Facebook, LinkedIn, Twitter, etc. A wide selection of journals (inclusive of 9 subjects, more than 200 journals)

Providing 24-hour high-quality service

User-friendly online submission system

Fair and swift peer-review system

Efficient typesetting and proofreading procedure

Display of the result of downloads and visits, as well as the number of cited articles Maximum dissemination of your research work

Submit your manuscript at: http://papersubmission.scirp.org/

Or contact ijcm@scirp.org 